\title{
Showbiz Legislation in Italy and Albania: Comparative Analysis
}

\author{
Arjan Vasjari PhD \\ Dariel Sina PhD
}

European University of Tirana

Doi:10.5901/ajis.2013.v2n2p415

\begin{abstract}
Legal decision-making in Albania as well as in EU countries (including Italy) concerning culture and showbiz is relatively recent. The Treaty of Rome did not contain in fact any specific chapter or paragraph for treating cultural policies. Only in the Treaty preamble was indirectly suggested that the culture is a unifying element and promoter of socio-economic development.In Albania, due to known causes, decision-making concerning culture and showbiz has been of secondary, let alone tertiary level. Shifting from a centralised and ideologised model of cultural policy that had nothing to do with the free market rules to a new model was traumatic. The absence of mentality, in particular, as well as the legal vacuum made it for many artists and individuals working in the domain of arts, the new reality to be experienced as a failure. Italy could offer a model to follow but the terrain for having it adapted, and moreover to have it applied was not one of the most compatible with this new wave of changes. Nevertheless, the great number of foreign artists having come to Albania (most of them from Italy) influenced not only the transformation of the etatist mentality of art-making and culture but also encouraged lawmakers and artists for drafting a modern legal framework on showbiz. Thus, acknowledging the great cultural and social value of the showbiz, the main objective is the verification of the current normative stage of this sector in both countries, highlighting the differences, similarities, possible legal gaps, with the aim of formulating proposals and fulfil legal gaps, considering the need for approximating the Albanian legislation to the principles of community law. This is the objective this comparative analysis aims to achieve.
\end{abstract}

\section{Gli imprenditori dello spettacolo in Italia}

In linea di principio si puo affermare che: l'azienda di spettacolo e un'azienda come tutte le altre.

Tuttavia, se l'affinita con altri settori economici e merceologici è riscontrabile, ad esempio, nella necessita del possesso di un numero di partita Iva, nell'obbligo contributivo nei confronti dei dipendenti e nell'opportunita della vendita del bene/servizio prodotto, ecc., le somiglianze si fermano piu o meno qui, poiche vengono in rilievo le specificita economiche dei mestieri della comunicazione sociale in generale e dello spettacolo in particolare.

L'attivita di spettacolo comporta una motivazione personale ed una vocazione espressiva assimilabile a quella di pochi altri mestieri. Quello dello spettacolo, come del resto altri mestieri nell'ambito della comunicazione sociale, fonda la sua sopravvivenza su un sistema di provvidenze pubbliche la cui organizzazione e logica d'uso non e di facile lettura.

Di fatto non e prevedibile la sopravvivenza economica del teatro, ad esempio, al di fuori di questo sistema, ne vale ricorrere ad esempi di imprenditoria teatrale che invece smentiscono queste affermazioni, perche questi esempi sono pochissimi e si giustificano proprio perchè operano in parallelo e in concorrenza ad un sistema definito, con qualche improprietà, "pubblicamente assistito".

D'altro canto, il teatro e la comunicazione sociale in generale, sono attivita eminentemente private ma con un rilievo sociale, quindi pubblico, rimarchevole. Non sarebbe altrimenti pensabile l'intervento dei pubblici poteri a sostegno di una privata attivita dell'ingegno umano se non in questa chiave.

L'economia dello spettacolo dal vivo fonda la sua esistenza su un sistema di provvidenze pubbliche che trova valore nel riconoscimento del valore sociale e culturale delle sue discipline.

Se è fuor di dubbio che l'economia dello spettacolo e la sua amministrazione, come tutte, si basa su un sistema di fonti ed impieghi delle risorse, le provvidenze pubbliche costituiscono la gran parte delle possibili fonti che le attivita dello spettacolo dal vivo possono prevedere. Non si vuole escludere che il settore dello spettacolo dal vivo abbia la capacita di remunerare l'attivita attraverso i convenzionali passaggi dell'economia di mercato (sbigliettamento, sponsorizzazioni private, merchandising ecc.), ma questa attiene principalmente all'operativita di strutture di spettacolo stabili, ossia operanti nel lungo periodo su di un territorio sempre uguale, con la possibilità quindi di fidelizzare il pubblicoe di attrarre 
risorse private in relazione alla specificità (sociale, culturale, di reddito ecc.) del pubblico fidelizzato. Se il teatro, infatti, dovesse trovare le risorse per la sua sopravvivenza esclusivamente sul libero mercato della vendita del prodotto, il costo del biglietto d'ingresso nelle sale dovrebbe essere altissimo e quindi automaticamente verrebbe esclusa la possibilità, da parte della collettivita, di godere di questa forma della comunicazione sociale. II ruolo dell'assistenza pubblica al teatro, infatti, consiste nell'integrazione del reale prezzo di mercato del biglietto d'ingresso rispetto al prezzo al quale esso e venduto realmente al botteghino. La vendita del prodotto teatrale non consente una remunerazione tale da coprire i suoi costi di produzione, pertanto l'impresa di spettacolo e in moltissimi casi una impresa a priori passiva economicamente. L'attivita di produzione ed organizzazione dello spettacolo dal vivo, per arrivare a prevedere qualsiasi pratica di natura amministrativa, deve, come si dice, "creare impresa": ne e pensabile, del resto, che una professione cosi particolare e complessa come questa possa esplicarsi al di fuori delle logiche e dell'organizzazione di impresa. Per organizzare e produrre spettacolo, sara necessario disporre di una "azienda" all'interno della quale si manifestino e vengano coordinate ed amministrate tutte le competenze necessarie per giungere alia progettazione, elaborazione culturale, creazione, gestione, promozione e vendita del prodotto spettacolare.

Non e necessario che si tratti di una "azienda" autonoma o privata: puo benissimo essere il "settore" di una impresa turistica, nel quale si concentra lo sforzo di elaborazione di marketing territoriale di un tour operator; oppure puo essere il "settore" di una pubblica Amministrazione; oppure, ancora, realmente una "azienda" autonoma e privata che opera a sostegno delle strategie di marketing turistico- territoriale che utilizza l'attivita di spettacolo come veicolo promozionale del territorio; potra essere anche una "azienda" che fornisce servizi di consulenza progettuale ed organizzativa al comparto turistico e non solo e potra essere molte cose ancora. L'azienda dovra avere caratteristiche particolari che la renderanno disomogenea rispetto a qualsiasi altra, in considerazione della natura, dei prodotti o servizi che deve essere in grado di esprimere, delle professionalità e competenze necessarie, delle modalità di coordinamento ed organizzazione. Lo spettacolo dal vivo in generale ed il teatro in particolare consistono, infatti, nell'attivazione di un rapporto tra gruppi distinti di uomini (gli attori e gli spettatori) che si realizza grazie al tramite di un prodotto artistico, che rappresenta poi la dimensione spazio - temporale entro la quale questo rapporto si crea.

Volendo procedere ad un'analisi delle modalita di esercizio deM'impresa dello spettacolo, si puo partire dalla definizione in base alia quale: imprenditore dello spettacolo $\mathrm{cd}$. impresario o produttore è colui che esercita professionalmente un'attivita economica rivolta alla produzione dello spettacolo e dei servizi connessi al fine di trarne guadagno. Pertanto, sono qualificabili imprenditori dello spettacolo: i titolari o i gestori di locali pubblici; i produttori cinematografici; le compagnie e gli impresari di spettacolo di prosa, ovvero, chiunque assume alle proprie dipendenze personale artistico e tecnico per la produzione e la realizzazione di spettacoli da tenersi in luoghi pubblici o privati e fornisce servizi collegati ad essi (affitto attrezzature, palchi o altro materiale necessario alio spettacolo).

II legislatore ha messo a disposizione degli operatori privati e pubblici una molteplicita di strumenti gestionali. Le forme di gestioni possibili per l'operatore privato sono differenti a seconda che egli si proponga o meno di conseguire degli utili. E' possibile distinguere a tal proposito:

1) l'impresa individuale, le societa di persona, di capitali e cooperative sono le forme di gestione idonee per il perseguimento dello scopo lucrativo (settore profit);

2) le formule associative non imprenditoriali, quali le associazioni con o senza personalità giuridica, i comitati, le fondazioni, sono le tipologie che si propongono di perseguire finalita diverse da quelle lucrative (settore non profit).

Per quel che riguarda l'operatore pubblico, per lo più territoriale, l'ordinamento italiano prevede le seguenti forme di gestione:

1) gestione diretta o in economia per mezzo di strutture organizzative interne all'ente locale;

2) gestione indiretta tramite affidamento diretto ad istituzioni, aziende speciali, associazioni e fondazioni costituite o partecipate, societa in house providing o a partecipazione pubblica maggioritaria;

3) gestione indiretta tramite concessione a terzi.

Una delle forme di gestione che meglio si adatta alle particolari esigenze del settore dello spettacolo e la gestione tramite istituzione. Con questa forma di gestione, l'ente locale istituisce al proprio interno un'entita dotata di autonomia sia gestionale che organizzativa, ma soprattutto contabile, pur priva di personality giuridica, alia quale affida la gestione di un settore di attivita. Questa entita deve essere istituita con l'approvazione, in seno al Consiglio Comunale o Provinciale, di un apposito regolamento per l'istituzione e per il funzionamento dell'ente. Trattandosi di un organismo strumentale rispetto all'ente locale, esso non è dotato di una propria autonomia, tant'è che usufruisce della stessa partita Iva dell'ente locale. Sicuramente rappresenta una forma di gestione molto utilizzata dai Comuni, ma l'aspetto piu critico e 
rappresentato dal fatto che il peso di questa istituzione grava in toto sul bilancio dell'ente locale, inoltre piuttosto scomodo appare il ricorso alle risorse umane interne all'ente. Ne deriva che la partecipazione del privato si riduce a forme di sponsorizzazioni erogate solo con l'obiettivo che ci sia un ritorno d'immagine.

La gestione in economia, invece, rappresenta una forma di gestione diretta del servizio pubblico e quindi vengono utilizzate le strutture organizzative interne all'amministrazione stessa, che generalmente fanno capo all'assessorato alia cultura. I punti deboli di questa forma di gestione sono riconducibili al fatto che vi e un'eccessiva burocratizzazione della gestione, con previsione di tempi molto lunghi, soprattutto per l'affidamento dei servizi. Inoltre, la poca trasparenza della gestione, a causa del fatto che la gestione in economia manca di un bilancio autonomo per l'attivita da svolgere, unitamente al fatto che le risorse umane da impiegare devono essere interne all'ente, fa sì che il ricorso a questa forma di gestione non sia auspicabile, anche se sicuramente un aspetto a suo favore e dovuto al fatto che l'ente locale non deve sobbarcarsi di ulteriori costi aggiuntivi per la creazione di un apparato esterno che gestisca l'attivita.

Altra forma di gestione e quella tramite affidamento diretto ad azienda speciale, attraverso la quale, l'ente locale crea un'entita diversa da se stesso, dotata di personalità giuridica ed autonomia imprenditoriale, gestionale, organizzativa e di bilancio, alla quale affida la gestione di un servizio.

Viene costituita con l'approvazione di uno Statuto ed un Regolamento per il suo funzionamento da parte dell'ente locale cui fa riferimento. L'azienda speciale e dotata di una propria partita Iva, redige un proprio bilancio tenendo una contabilita autonoma. Ha l'obbligo di iscriversi al Registro delle imprese.

Le risorse da impiegare al suo interno devono essere reperite al di fuori dell'organico dell'ente cui fa capo.

Sono possibili altre forme di affidamenti diretti: ad associazione costituita o partecipata dall'ente locale, a fondazione costituita o partecipata dall'ente locale, a s.r.l. o s.p.a. a capitale interamente pubblico.

Presuppone, al contrario, l'espletamento di una procedura ad evidenza pubblica, l'affidamento tramite gara a s.r.l. o s.p.a. partecipate in misura prevalente dall'ente locale. E' una modalita di gestione indiretta del servizio, nel senso che l'ente locale, se pure detentore della maggioranza del capitale di una societa operante nel settore della gestione dei servizi pubblici privi di rilevanza economica, deve espletare, un'apposita gara per individuare la societa cui affidare la gestione del servizio pubblico.

Nella concessione a terzi, invece, l'ente locale affida totalmente o parzialmente la gestione del servizio pubblico a terzi, quali associazioni culturali locali, compagnie di spettacolo,pro loco, con l'obiettivo di assicurare un adeguato livello di valorizzazione dei beni culturali, previa valutazione comparativa della sostenibilita economica- finanziaria del progetto.

Con la valutazione di cui sopra, l'ente pubblico deve dimostrare che l'esternalizzazione risulta conveniente rispetto ad una gestione diretta, in termini di efficienza ed efficacia, con riferimento a obiettivi, mezzi, metodi e tempi. Quindi con questa forma di gestione la titolarità del servizio e le attività connesse restano in capo all'ente concedente, mentre l'esercizio del servizio viene trasferito al concessionario privato. I rapporti tra concedente e concessionario sono regolati dal contratto di servizio. Eventuali inadempimenti del concessionario determinano la risoluzione del rapporto concessorio. Particolare attenzione merita il consorzio. Si tratta di una forma di gestione indiretta con affidamento diretto, creata per la volonta di piu soggetti pubblici, anche diversi dagli enti locali, che intendono operare congiuntamente per offrire uno o piu servizi nei territori di riferimento, o per la gestione associata di funzioni. I consorzi sono enti con personalità giuridica. La partecipazione dei privati è esclusa. Si costituiscono grazie all'approvazione della Convenzione e dello Statuto da parte di tutti i Consigli degli enti interessati, attraverso il conferimento di una quota di capitale.

\section{Gli adempimenti e l'iter burocratico nella legislazione italiana}

Qualunque sia la forma giuridica adottata, qualsiasi impresa di spettacolo, che sia di produzione, di organizzazione o di ospitalita, in qualsiasi forma sia organizzata, e tenuta ad immatricolarsi presso l'Ente Nazionale di Previdenza e Assistenza per i Lavoratori dello Spettacolo.

L'immatricolazione consiste nella presentazione da parte del legale rappresentante della società, presso la sede territoriale di competenza o all'ufficio Siae.

Tutte le imprese di spettacolo che occupano lavoratori autonomi, subordinati 0 assimilati, quali lavoratori a progetto, i collaboratori coordinati e continuativi, devono immatricolarsi anche presso l'Inps, al fine di versare i contributi previdenziali per la maternita, disoccupazione e assegni familiari. Piu specificatamente l'imprenditore deve costituire presso I'Inps due diverse posizioni contributive: una destinata ai versamenti contributivi previdenziali dei lavoratori subordinati; l'altra destinata ai versamenti contributivi previdenziali di una serie di lavoratori indicati tassativamente dalla normativa, per i quali si dice che opera la gestione separata Inps. Si tratta della situazione in cui il lavoratore ha gia una 
propria copertura previdenziale o perche dipendente di altro ente o perche imprenditore oppure perche il lavoratore e un libero professionista privo di una propria cassa di previdenza o di tutte le situazioni di lavoro autonomo parasubordinato del tipo lavoro a progetto e collaborazione coordinata e continuativa.

L'imprenditore di spettacolo, ogni qualvolta decida di impiegare dei lavoratori, deve assolvere a determinati obblighi. L'Enpals, I'Ente Nazionale di Previdenza e Assistenza per i lavoratori dello Spettacolo, con la circolare n. 21 del 04/06/02, ha fornito un quadro normativo completo in tale materia, al fine di uniformare la prassi su tutto il territorio nazionale.

Grazie alla convenzione Siae/Enpals la procedura per la richiesta del certificato e molto snella, visto che diverse associazioni operanti nel settore dello spettacolo dal vivo, hanno stipulato convenzioni telematiche con la Siae. II rilascio del certificato avviene nel giro di breve tempo, talvolta anche contestualmente all'inizio dell'evento per il quale e richiesto.

Per quanto riguarda le imprese straniere che operano nel nostro territorio, esse devono richiedere il certificato di agibilita all'Enpals, indipendentemente dal luogo dove eseguono i versamenti.

In tal caso l'Enpals rilascia loro il certificato con esenzione contributiva e cioe a titolo gratuito, previa esibizione dei documenti da cui si evince l'avvenuto versamento: diversamente il certificato viene rilasciato previo versamento anticipato della contribuzione. Non sempre le imprese di spettacolo scritturano direttamente i lavoratori iscritti: nella realta accade spesso che l'imprenditore stipuli contratti con associazioni, fondazioni, societa cooperative che impiegano i lavoratori iscritti. Ovviamente, in quest'ultimo caso, graverà su dette associazioni, fondazioni, etc. assolvere agli oneri contributivi.

Vi sono, tuttavia, dei casi in cui il datore e esonerato dai versamenti Enpals e dalla richiesta del certificato di agibilita, casi tassativamente previsti dalla Circolare n. 21/2002. Cio avviene per i saggi di danza o per i saggi di altre arti, effettuati da bambini e giovani frequentanti corsi didattici, oppure per manifestazioni organizzate ai fini socio- educativi da oratori, associazioni con riconoscimento ecclesiale o associazioni religiose, da associazioni di promozione sociale e da cooperative sociali.

Tale esenzione ricorre anche nel caso in cui l'organizzatore dello spettacolo impegni formazioni dilettantistiche 0 amatoriali (complessi bandistici comunali, gruppi folkloristici, compagnie teatrali amatoriali etc..) allo scopo di divertimento elo per tramandare tradizioni popolari e folkloristiche, senza perseguire alcuna forma di remunerazione, ma a titolo gratuito. Nella stessa circolare sono anche previsti i casi in cui il certificato di agibilita e rilasciato a titolo gratuito, nel senso che il datore di lavoro deve comunque richiederlo, ma la richiesta non comporta alcun versamento contributivo a favore del lavoratore. Tale situazione si verifica ogniqualvolta la manifestazione artistica si svolga a scopo benefico, sociale o solidaristico e gli eventuali ricavi, detratte le spese di allestimento e di organizzazione, siano integralmente devoluti alle finalita per cui la manifestazione si e svolta. I lavoratori, in questo caso, non devono percepire alcun compenso per la prestazione svolta e l'organizzatore e tenuto a rilasciare un attestato sulla natura benefica dell'evento dal quale si evinca l'assenza di compensi per i lavoratori. Un'ulteriore fattispecie di esenzione di certificato, riguarda l'occupazione di lavoratori di nazionalita straniera che certifichino di aver adempiuto agli oneri contributivi nel paese di origine $\mathrm{o}$, ancora, nel caso in cui vengano impiegati complessi stranieri che effettuano spettacoli nell'ambito di scambi cultural) internazionali, ricevendo solo un rimborso per le spese di trasferimento e soggiorno sostenute nel nostro paese. In questi ultimi due casi spetta all'ente italiano che riceve la prestazione richiedere il certificato di agibilita Enpals. II Testo Unico delle Leggi di Pubblica Sicurezza ed il relativo regolamento di esecuzione prevedono, inoltre, che l'organizzatore di attivita di spettacolo e intrattenimento in luogo pubblico, aperto al pubblico o esposto al pubblico, debba essere munito della licenza di esercizio, detta anche licenza di pubblica sicurezza.

Per quanto riguarda la differenza tra spettacolo ed intrattenimento, a tal proposito occorre far riferimento alia Circolare ministeriale 165/2000, per mezzo della quale, il Ministero degli Interni chiarisce che "per intrattenimento si intende ciò che è cagione di divertimento, insito, quindi, nel concetto e partecipare. Lo spettacolo, invece, è caratterizzato dal concetto di rappresentazione, perciò riguarda l'assetto statico del fenomeno, di presenza ad un fatto 0 ad un avvenimento. In sintesi, si può affermare che, mentre, in generale, l'intrattenimento implica la partecipazione attiva all'evento, lo spettacolo comporta prevaientemente una partecipazione passiva, lo spettatore assiste al fenomeno, guarda l'evento cosi come gli e rappresentato."

\section{II finanziamento delle attivita' di spettacolo in Italia}

L'incertezza finanziaria in cui versa il settore dello spettacolo, incapace di sostenersi con risorse proprie, unitamente al fatto che nell'ultimo decennio vi e stato un significativo calo dell'investimento di risorse pubbliche nel settore della cultura 
in generale, ha contribuito a far si che il comparto dello spettacolo si sia avvalso di fonti alternative di sostentamento, in grado di sopperire alle mancanze delle istituzioni pubbliche. sintetizzate:

Preliminarmente ricordiamo che le principali fonti di entrata nel settore dello spettacolo possono essere cosi

- $\quad$ risorse economiche proprie dell'impresa di spettacolo, quali l'apporto di capitale e gli utili accumulati in esercizi precedenti;

- contributi e sovvenzioni dell'Unione Europea, dello Stato e delle Regioni, delle Province e dei Comuni;

- erogazioni liberali provenienti spesso da fondazioni bancarie o da altre istituzioni culturali;

- i ricavi derivanti dalla rappresentazione e dalla distribuzione del prodotto ossia gli incassi ed i cachet;

- i ricavi da sponsorizzazioni.

E' su quest'ultimo aspetto che e opportuno focalizzare l'attenzione. II contratto di sponsorizzazione e quel contratto mediante il quale un'impresa (sponsor), al fine di pubblicizzare il proprio nome 0 la propria immagine 0 determinati prodotti, viene a finanziare eventi sportivi, singoli atleti, squadre ottenendo quale corrispettivo, l'assunzione di un obbligo di reclamizzazione.Lo sponsor e quindi un soggetto privato, persona fisica o giuridica, disposto a investire un determinato corrispettivo in cambio di pubblicita, lo sponsorizzato e, invece, quel soggetto privato o pubblico, associazione o impresa, ente, che organizza e gestisce l'evento spettacolo e che, a fronte dell'impegno economico assunto dallo sponsor, si impegna a diffondere un messaggio pubblicitario in varie forme.

Seppure libera nella forma, a meno che parte contraente non sia un ente pubblico, nel qual caso la forma e prevista obbligatoriamente per iscritto, il contratto di sponsorizzazione e un contratto consensuale, a prestazioni corrispettive ed, infine, a carattere oneroso. Con la sottoscrizione del contratto si determina il sorgere di una obbligazione di mezzi ma non un'obbligazione di risultato, ragione per cui lo sponsorizzato è tenuto a fornire allo sponsor solo la visibilita contrattata, attraverso la citazione dello sponsor nei comunicati stampa, apponendo il logo su brochure e su altro materiale cartaceo da affiggere o distritrtiire, consentendo allo sponsor di presenziare durante le conferenze stampa. Sempre sullo sponsorizzato incombe l'obbligo di realizzare al meglio l'evento, con la dovuta diligenza, nella consapevolezza che non operando in modo corretto ed efficace, potrebbe arrecare un danno all'immagine dello sponsor.

Come gia innanzi accennato, salvo diversa pattuizione, con la sottoscrizione di un accordo di sponsorizzazione, non sorge in capo allo sponsorizzato alcun obbligo di risultato: quindi, nel caso di insoddisfazione dello sponsor, egli non sara esonerato dall'effettuare il versamento della somma o dei beni pattuiti in sede di contratto. Sotto l'aspetto fiscale, la sponsorizzazione costituisce un'operazione che rientra nel campo di applicazione IVA, in quanto, trattasi di "cessione di beni" o" prestazione di servizi".

Pertanto, lo sponsorizzato e tenuto, qualora sia in possesso di partita IVA, ad emettere regolare fattura, con applicazione dell'Iva al $20 \%$. Nel caso in cui lo sponsorizzato sia privo di partita IVA, come per esempio nel caso di un'associazione che non svolge regolarmente attivita commerciale, dovrà essere emessa una ricevuta che attesti l'avvenuto incasso della somma pattuita. Fattura e ricevuta dovranno essere emesse anche nel caso in cui lo sponsor, non corrisponde una somma di denaro, ma si impegna ad una prestazione di dare (fornire un bene), o ad una prestazione di fare (compimento di una determinata attivita). Fattura e ricevuta rilasciata dallo sponsorizzato, consentono allo sponsor di dedurre i costi sostenuti relativamente alia sponsorizzazione.

Per quanto concerne l'utilizzo del contratto di sponsorizzazione da parte della Pubblica Amministrazione, tale possibilità viene riconosciuta in virtu della Legge 27 dicembre 1997 n.449, che all'art. 43 così dispone: "al fine di favorire l'innovazione dell'organizzazione amministrativa e di realizzare maggiori economie, nonche una migliore qualita dei servizi prestati, le pubbliche amministrazioni possono stipulare contratti di sponsorizzazione ed accordi di collaborazione con soggetti privati ed associazioni, senza fini di lucro, costituite con atto notarile".

L'ammissibilita della stipula del contratto in esame dipende dal ricorrere di alcune condizioni individuate dal legislature, quali il perseguimento di interessi pubblici, l'esclusione di conflitto di interessi tra attività pubblica e quella privata e conseguimento di un risparmio di spesa rispetto agli stanziamenti disposti.

La volontà di procedere alla stipula di un contratto di sponsorizzazione con la pubblica amministrazione deve avvenire nel rispetto delle regole pubblicistiche, e per la scelta del contraente devono essere utilizzate le procedure di evidenza pubblica. A tal proposito ogni ente deve o dovrebbe essere dotato di apposito regolamento sulle sponsorizzazioni, approvato dall'organo competente. L'avvio dell'attivita di procacciamento delle sponsorizzazioni nella generalità dei casi dovrebbe coincidere con la pubblicazione di un bando, attraverso il quale l'ente invita i potenziali sponsor alla trattativa. Cio puo non accadere nel caso in cui, trattandosi di una sponsorizzazione di valore piuttosto modico, l'ente deliberi di procedere a trattativa diretta. 
Diversa dalla fattispecie contrattuale sin qui esaminata, e l'erogazione liberale. Essa consiste sempre in erogazioni di somme di denaro e non possono consistere in forniture di beni e di servizi. La corresponsione di dette somme da parte di un'impresa individuale o collettiva avviene al di fuori di un rapporto di corrispettivita. Ragion per cui il beneficiario non è tenuto ad impegnarsi ad alcuna attivita promozionale, ne è tenuto ad offrire al soggetto erogante alcun ritorno di immagine. Di tal che non ricorrono, nel caso di erogazione liberale i presupposti per l'applicazione dell'IVA: quindi il beneficiario della erogazione non dovra emettere alcuna fattura o ricevuta di pagamento. Da quanto sin qui detto, appare difficile che nella realta esista un soggetto disponibile a erogare somme di denaro per un evento spettacolare, senza poter conseguire tuttavia alcun vantaggio, nemmeno da un punto di vista di ritorno di immagine.

\section{La legislazione albanese}

Anche la legislazione albanese individua e disciplina forme di gestione e produzione di spettacoli sia per gli operatori pubblici che per quelli privati.

I principali operatori pubblici sono le istituzioni pubbliche locali di arte scenica le quali sono istituzioni finanziarie che vengono costituite o trasformate secondo la legislazione vigente, su iniziativa e volonta dell'autorita locale dalla quale dipendono. Lo statuto dell'istituzione pubblica locale di arte scenica viene proposto dal Comitato Artistico e approvato dal Consiglio di unità della rispettiva autorita locale.

La composizione del Comitato Artistico dell'istituzione pubblica locale è approvato dal Consiglio di unità dell'autorita locale. I membri del comitato hanno un mandato di tre anni, rinnovabile. L'istituzione pubblica locale di arte scenica ha la sua sede presso l'unita amministrativa locale. Le fonti di finanziamento dell'istituzione pubblica locale di arte scenica sono:

- i fondi della finanziaria per le autorita locali a sostegno delle spese amministrative

- $\quad$ il bilancio dell'ente locale per la cultura

- le entrate procurate dai progetti vincitori dei fondi del Centro Nazionale di Arte Scenica

- le entrate procurate dalla vendita dell'opera scenica

- l'incasso dei biglietti, e dei servizi che l'istituzione assicura ai terzi

- le sponsorizzazioni e donazioni.

II Direttore dell'istituzione pubblica locale di arte scenica e nominato dal Consiglio dell'ente locale, selezionato tra due candidature proposte dal sindaco. Le procedure di nomina sono stabilite dallo Statuto approvato dal Consiglio dell'unita locale. I rapporti giuridici di lavoro nell'istituzione pubblica locale di arte scenica sono regolati dal Codice del Lavoro della Repubblica d'Albania, nonche dallo Statuto e Regolamento interno.

Per "Teatro metropolitano" si intende lo status particolare conferito ad un'istituzione pubblica locale in base alla storia, al particolare valore artistico ed al contributo dato alia cultura nazionale. Le procedure, le condizioni ed i criteri per il conferimento di status 'Teatro metropolitano' vengono stabilite con delibera del Consiglio dei Ministri. I soggetti privati di arte scenica operano secondo la legge nr.7638, dt.19.11.1992 "Per le societa- commerciali" e la legge nr.8788, dt.07.05.2001 "Per le organizzazioni non lucrative". Sono tenuti a registrarsi presso Centro Nazionale di Arte Scenica e ad osservare la legislazione fiscale vigente, onde poter usufruire dei finanziamenti in favore dei progetti privati scenici messi a disposizione dal fondo pubblico di arte scenica.

Le forme societarie previste dalla legislazione vigente sono essenzialmente quattro: societa in nome collettivo, societa in accomandita semplice, societa a responsabilita limitata e societa per azioni, la cui regolamentazione e in linea con la normativa italiana. Esistono, inoltre, altre tipologie di societa commerciali (Fondi di Investimento, Societa di Credito e Risparmio, Societa Cooperative) la cui disciplina e demandata a leggi speciali.

Le societa vengono registrate nell'ufficio del Registro commerciale ubicato presso il tribunale distrettuale di Tirana. II costo medio per la registrazione, comprensivo di costi amministrativi e spese notarili, e di circa 1000 euro. A seguito della registrazione alla società viene rilasciata una licenza permanente per avviare la propria attivita. Successivamente all'iscrizione nel registro commerciale occorre richiedere alle autorita fiscali una licenza generale di commercio (da non contendere con la licenza amministrativa) ed un numero di identificazione fiscale (tale licenza va annualmente rinnovata). Tornando all'arte, come è noto, l'Albania finanzia con fondi pubblici i progetti artistici. La misura del compenso del valore artistico nell'arte scenica spettante al progetto e determinato con delibera del Consiglio dei Ministri.

Le condizioni, i criteri e la misura del compenso per i risultati conseguiti nei concorsi internazionali di arte scenica si determinano con delibera del Consiglio dei Ministri. La legislazione regolamenta sia il contratto di commissione di un'opera che il contratto di produzione degli spettacoli. II contratto di commissione di un'opera deve regolamentare il 
termine per la consegna e le condizioni di accettazione da parte del committente, il quale ha la facoltà di recedere dal contratto se l'opera non soddisfa le condizioni contrattuali. Quello per la produzione dello spettacolo teatrale e/o musicale, invece, ha per oggetto la trasmissione da parte dell'autore o del titolare del diritto d'autore, dei diritti di rappresentazione al pubblico dell'opera letteraria, drammatica, drammatico-musicale, coreografica o di un pantomima, al produttore dello spettacolo. II contratto, concluso in forma scritta, spiega efficacia a seguito della registrazione e certificazione presso l'Ufficio Albanese per i Diritti d'Autore e si conclude allo scadere del termine concordato e del numero prestabilito di spettacoli in pubblico.

Nel contratto occorre stabilire il periodo di tempo in cui si realizzera lo spettacolo, il numero delle rappresentazioni, la clausola di esclusiva o meno della trasmissione dei diritti, le condizioni di verifica degli incassi e le modalita per la determinazione del corrispettivo dell'autore o del titolare dei diritti d'autore. Se nel contratto non è previsto diversamente, la mancata esecuzione dello spettacolo per due anni consecutivi, conferisce all'autore 0 al titolare dei diritti d'autore, il diritto di annullare il contratto, di chiedere il risarcimento dei danni causati ed il mancato profitto, in conformita con le disposizioni di legge in vigore. L'acquirente del diritto per la rappresentazione dello spettacolo teatrale e/o musicale non ha la facoltà di trasferire i diritti a terzi senza l'approvazione scritta dell'autore o del titolare dei diritti d'autore, egli, inoltre, è obbligato $\mathrm{a}$ :

- $\quad$ consentire all'autore 0 al titolare dei diritti d'autore di controllare la rappresentazione dell'opera;

- $\quad$ assicurare il rispetto dell'applicazione delle condizioni tecniche per la rappresentazione dell'opera;

- $\quad$ consegnare all'autore $o$ al titolare dei diritti d'autore il programma, il manifesto, il materiale stampato cosi come le recensioni del pubblico relativo a spettacolo;

- $\quad$ comunicare per iscritto il numero degli spettacoli realizzati in pubblico, la situazione degli incassi di ogni spettacolo, su richiesta dell'autore o titolare dei diritti d'autore.

Nel caso in cui l'acquirente del diritto per la rappresentazione dell'opera non esegua in pubblico l'opera nel periodo concordato, all'autore 0 al titolare del diritto d'autore e riconosciuto il diritto di recedere unilateralmente dal contratto, chiedendo il risarcimento per il danno causato e il mancato guadagno secondo le disposizioni della legge in vigore. Ogni accordo per il trasferimento dei diritti patrimoniali in forma esclusiva viene depositato, registrato e certificato presso I'Ufficio Albanese per i Diritti d'Autore.

\section{Analisi comparata}

Le problematiche relative all'impresa e lo spettacolo appaiono comuni sia in Italia che in Albania: in entrambi gli ordinamenti e prevista la possibilità per operatori pubblici ed operatori privati di "fare spettacolo"; in entrambi gli ordinamenti, l'economia dello spettacolo dal vivo fonda la sua esistenza su un sistema di provvidenze pubbliche che trova giustificazione nel riconoscimento del valore sociale e culturale delle sue discipline.

L'attenzione del legislatore, pertanto, è focalizzata non tanto a prevedere e regolamentare forme giuridiche specifiche, quanto piuttosto a disciplinare le modalita di accesso ai finanziamenti pubblici da parte degli operatori, ciò in considerazione delle peculiarità del settore dello spettacolo dal vivo.

La differenza principale può essere, invece, individuata nel potere che viene riconosciuto in Albania allo Stato di determinare la misura del compenso del valore artistico dell'opera scenica. Probabilmente la sopravvivenza di tale potere è da giustificare con il lento processo di democratizzazione che sta vivendo l'Albania e che non puo dirsi ancora completato.

Degno di nota e, tuttavia, il fatto che anche in Albania cosi come in Italia una delle principali fonti di finanziamento dello spettacolo sono le sponsorizzazioni dei privati, segno del graduale, seppur lento e difficile, affermarsi di un'economia di mercato in luogo di quella di Stato.

\section{Bibliografia}

Legislazione dello spettacolo dal vivo,Anna Popi,Edizione Simone 2007

Risoluzione del PE del 7 giugno 2007,Approvazione dello Statuto Sociale degli Artisti

Dossier tematico "La normativa sullo spettacolo dal vivo" a cura della Fondazione Rosselli

Democrazia locale in transizione,Arolda Elbasani su Balcani Cooperazione:le relazioni territoriali tra Italia e Sud EstEuropa,10.10.2007 
"Le risorse destinate allo spettacolo dal vivo e al cinema:lo strumento fiscale" di Maria Cecilia Fregni,su Aedon Rivista di diritto ed arti on line n.3/2007

Le nuove norme a tutela del diritto d'autore nella normativa nazionale e comunitaria,Barbara Betteli

Codice di Lavoro Albanese

Legge n.7638 del 19.12.1992 "Per le societa commerciali"

Legge n7986 del 13 settembre 1995 "Sull'ispetorato del Lavoro dello Stato"

Legge n.8788 del 07.05.2001 "Per le organizzazioni non lucrative"

Legge dell'arte scenica,2006

MTKRS,La legge per l'arte e la cultura,nr.10352,18.11.2010, 